\title{
Assessing Pre-Service Teachers' Offensive Basketball Game Performance: A Validation Study 對體育教學專業生籃球進攻技戰術测評的效度研究
}

\author{
Weiyun CHEN Kristin HENDRICKS \\ School of Kinesiology, \\ University of Michigan, USA \\ 陳蔚雲 克利斯梯, 翰狄克 \\ 美國密歇根大學人體科學學院
}

\begin{abstract}
迎
Abstract

The purpose of this study was to design and validate the Basketball Offensive Game Performance Instrument (BOGPI) that assessed an individual player's offensive game performance competency in basketball while watching a videotaped game play. Twelve physical education teacher education (PETE) students playing two 10-minute, 3 vs. 3 basketball games were videotaped at end of a basketball unit in a secondary methods course. Two investigators independently coded each player's offensive game behaviors with the BOGPI. The results of the experts' judgment, the independent t-tests, and the inter-rater reliability indicated that the BOGPI was a valid and reliable instrument for assessing the pre-service teachers' basketball offensive game ability.
\end{abstract}

\section{摘 要}

本研究目的旨在制定 “籃球進攻技戰術能力測評表” 並對其信度與效度進行檢驗。通過觀看比賽錄影, 利用測評表對每位 隊員的籃球進攻能力進行測評。在教法課籃球單元結束之時，研究人員對 12 名教學專業生參加 2 場 3 打 3 教學比賽錄了相。然 後, 兩名研究人員用該測評表對每位隊員的進攻比賽能力做了測評。專家鑒定, t-檢驗, 及測評人員之間信度的結果表明該測評 表具備可信性及有效性。

Key words: Game Performance, Assessment, Offensive Game Ability

\section{Introduction}

With an increasing application of the tactical games approach to teaching games over the past two decades, improving students' game performance competency is one of the ultimate goals of game learning and teaching (Gréhaigne, Godbout, \& Bouthier, 2001; Oslin, Mitchell, $\&$ Griffin, 1998). Game performance represents the intertwined process of a person's tactical awareness and knowledge, decision making ability, and skill execution in situated game contexts (Gréhaigne et al., 2001; Oslin et al., 1998). Scholars and teacher educators have recognized that the teacher's game performance competency is conducive to effectively teaching students game skills and tactics with a gradual increase of tactical complexity in situated and modified game contexts (McCullick, 2001; Siedentop, 2002). the Beginning Physical Education Teacher Standards (National Association for Sports and Physical Education (NASPE) (2009) explicitly describe that the pre-service teacher should be able to demonstrate competent movement performance and tactical concepts. Researchers stressed that the pre-service teacher's ability 
to skillfully perform the sports and physical activities is critical to becoming an effective physical education teacher (McCullick, 2001; Siedentop, 2002). This is because competency in performing various sport-related skills is directly linked to the effective demonstration of skills and tactics, efficient ways of teaching skills and tactics, and keen observation and evaluation of skills (McCullick, 2001; Siedentop, 2002).

The situated learning perspective views that the task should be situated in authentic and specific settings (Lave \& Wenger, 1991; Kirk \& MacPhail, 2002; Rovegno, 2006). Accordingly, a game performance assessment instrument should be designed for a specific game form. Although soccer, basketball, football, and team handball are classified into invasion games and share similar tactical concepts like gaining possession of the ball and attacking the goal, the interaction of the primary game rules, the number of field players, the size and dimensions of the field/court, and the specialized skills used for playing a specific sport makes each game context unique and different from one another. Like each sport having its specialized skills, each sport also has its specific ways to perform offthe-ball movements. Each game has its unique ways to handle the ball, move to open space, and interact with teammates and opponents. The game context of soccer is different from that of basketball (Kirk \& MacPhail, 2002; Rovegno, 2006). Therefore, the situated and specific nature of each game context demands designing a game specific assessment instrument.

The Game Performance Assessment Instrument (GPAI) designed by Oslin et al. (1998) has been widely used in secondary physical education and physical education teacher education. The GPAI was designed to assess students' abilities to make decisions, move appropriately, and execute skills across invasion, net, field, and target games. The GPAI identified and defined seven game components including Base, Adjust, Decisions Made, Skill Execution, Support, Cover, and Guard/Mark. As a flexible game performance assessment instrument, teachers and researchers may select any game components related to a specific game form like an invasion game form or a net game form for specific instructional purposes. For instance, if a teacher or a researcher wants to assess students' abilities to play invasion games, whether evaluating soccer or basketball, he/she may choose Decisions Made, Skill Execution, and Support, the three game components essential for offensive game play in either sport.
Memmert and Harvey (2008) pointed out that the GPAI ignored that the ways to handle similar tactical problems in basketball were different from other invasion games like soccer and hockey. Due to the fact that each sport within the invasion game form is context situated and specific, the broad definition of the game components on the GPAI might cause difficulty for teachers and researchers to objectively and reliably assess students' game behaviors in a specific invasion sport.

Memmert and Harvey (2008) noted that the GPAI coding category descriptions were rather general and subjective. For example, Griffin et al. (1997) described that the GPAI used either a two-point rating scale (e.g. use of appropriate/efficient or inappropriate/inefficient responses) or used a rubric-type 1-5 rating system to code players' individual game components during a 10-minute, 3 vs. 3 game play. However, the coding system of the GPAI did not describe the key information for independent observers/coders. For instance, the coding system of the GPAI did not include (a) the number of players to be observed and coded at a time throughout the game play, (b) when a coder should start to observe a player's game behaviors on each individual game component and when to stop the observation and then code the player's game behaviors, and (c) when the coder switched to observe the other player's game behaviors. Since the GPAI did not specify coding protocols related to specific and situated game context, the subjective and general coding system made it very challenging and difficult for independent coders to objectively judge and code if a player's specific game component was appropriate/ efficient or inappropriate/ inefficient.

Memmert and Harvey (2008) stated that although the comprehensive and flexible features of the GPAI have been widely recognized and the GPAI has been validated in selected invasion and net games, the current GPAI has limitations because of its broad definition of each individual game component and subjective coding protocols. There are pragmatic needs to modify the global features of the GPAI to make the game performance assessment criteria and coding protocols more adaptable to a particular game context and to define them as specifically as possible (Memmert \& Harvey, 2008). The purpose of this study was to design and validate the Basketball Offensive Game Performance Instrument (BOGPI). The BOGPI was designed as a teacher-assessment, selfassessment, and/or pee-assessment tool to assess an 
individual player's offensive game performance competency in basketball through observing the videotaped game play and/or during a live lesson in physical education teacher education. The specific objectives of this study were to (a) establish content validity of the BOGPI, (b) examine the construct validity of the instrument, and (c) determine the reliability of the instrument.

\section{Methods}

\section{Research Participants and Settings}

The participants were four male and eight female undergraduate students enrolled in one secondary methods course at a major university in the Midwest USA. They were junior and senior PETE majors with the average age of 21 years old and 2.27 standard deviation of age. Eleven participants were White and one participant was Asian. Of the participants, seven had varsity high school playing experience in basketball, while the other five participants did not play on a varsity basketball team before taking this course. The University Institutional Review Board approved the study. The participants signed an informed consent form.

The methods course was organized into four units: soccer, basketball, volleyball, and team hand ball. The course instructor used the tactical games approach (Mitchell et al., 2006) to teach the PETE students five basketball lessons, each lesson lasting two hours in length. Throughout the five basketball lessons, the PETE students learned the correct techniques of passing, dribbling, and shooting skills and the tactical purposes of applying the skills within the context of game positions and tactical game situations while following game rules. They also learned correct techniques for off-the-ball movement and tactical use of the movements, including (a) how to use different types of cuts to create open space and a passing lane, (b) how to set a screen and use the screen to create open space for attacking, and (c) how to relocate positions to support teammates.

\section{Development of the BOGPI}

The BOGPI was designed to provide teachers and researchers with an objective, reliable, and valid assessment instrument that assessed pre-service teachers' offensive game performance competency in basketball in authentic settings. The rationales for designing the BOGPI were to make each game component criteria specific to offensive game situations in basketball, to make sure that assessment coding protocols accommodate offensive basketball game situations, and to make the scoring system objective in assessing the pre-service teachers' game performance competency.

Definition of the game component. Two investigators used the three game components related to offensive basketball game situations, Skill Execution, Decision Making, and Support (Oslin et al., 1998), as three essential game dimensions in the BOGPI. They worked together to design, revise, test, and re-design the BOGPI.

The first investigator is the course instructor who has used the tactical games approach to teach the preservice teachers the secondary teaching methods course including basketball unit for 13 years. In addition, the first investigator played on a varsity basketball team in college and high school. The second investigator is a research assistant who earned the bachelor degree majoring in physical education teacher education and took the secondary methods course with the first investigator prior to being involved in this study. She played varsity high school basketball and also played three years of college basketball at the Division I and III level. In college, she was an instructor at summer basketball camps and yearly youth clinics provided by the women's basketball team. She has been coaching basketball for two years at the high school level as the freshman girls' coach and assistant varsity coach.

To ensure the three essential game dimensions can be more adaptable to a particular offensive game situation in basketball, the two investigators first identified subgame components within each game dimension in the BOGPI. For example, the sub-game components within the Skill Execution included three essential offensive skills in basketball: Dribbling, Passing, and Shooting. The sub-game components within the Decision Making dimension consisted of the Tactical Attempt of Dribbling, Passing, and Shooting. The sub-game components in the Support dimension contained Creating Space, Setting Screens, Reading the Defender, and Relocating to Support Teammates. 
Next, the two investigators used the situated learning perspective as the theoretical framework and Gréhaigne et al's (2001) two dimensions of game performance in team sports as the guidelines for defining each sub-game component in the BOGPI. As Gréhaigne et al. (2001) pointed out, the application of on-the-ball skills and offthe-ball movements involves a dynamic interaction between technical and tactical aspects. In situated game contexts, a player should apply a specific skill with a given tactical aim in order to solve given tactical problems. Therefore, the definition of each sub-game component should reflect the tactical and technical dimensions of a skill/movement.

Finally, the two investigators began to describe the definition of each sub-game component. To ensure each definition clearly reflected the technical and tactical aspects, they repeatedly used the cycle of discussing, testing, and re-visiting to revise the definition of each sub-game component. After numerous revisions of the definitions, the two investigators finalized the definition of each-sub game component in the BOGPI and the twopoint rating scale to help objectively assess a pre-service teacher's offensive game performance. (see Table 1).

\title{
Table 1. Definition of Each Sub-Game Component and Rating Scales in the BOGPI.
}

\author{
Game Dimension Definition of Each Game Component
}

Skill Execution:

1. Dribbling: Dribbles a ball when appropriate while changing pace and directions to maintain control of the ball.

2. Passing: Passes accurately when a teammate is open, has a good supporting position, or has the best shooting position.

3. Shooting: Shoots when getting open and scores a basket.
Decision Making:
1. Attempts to dribble to take on/beat defender, drive to the basket, or read situations.
2. Attempts to pass to set up a shot, move the ball, beat defender, or set up offense.
3. Attempts to shoot when in good position and wide open.

Support:
$\begin{aligned} & \text { 2. Reads defense and offense situations to effectively and appropriately use cuts or post up. } \\ & \text { 3. Reads the defender to effectively come off screens by using roll, pop out, curl, and/or fade appropriately } \\ & \text { 4. Reads defense/offense situations to effectively and appropriately relocate positions. }\end{aligned}$
Rating Scale:
"+" indicates that an individual player demonstrates the definition of each
individual game component.
"-" indicates that an individual player does not demonstrate the definition of each individual game component.

Coding protocols. An evaluator observed and recorded the presence or absence of a specified game behavior on each sub-game component with a tally mark when the observed players' team gained possession of the ball. The coding protocols were: (a) observing the targeted player's offensive game behaviors until a goal was scored, the ball went out of bounds, or the ball was intercepted; (b) coding the player's offensive game performance of each sub-game component using the event recording method; (c) re-watching the player's offensive game behaviors if necessary; (d) switching the observation and coding of the opponent's offensive game behaviors once there was a turnover in possession; (e) taking turns observing and coding the pair of individual players' offensive game behaviors throughout the 10-minute game play using the above procedures; (f) rewinding the DVD to the very beginning of the game; and (g) watching and coding the other two individual players' offensive game behaviors throughout the 10-minute game until all players' offensive game performance have been coded. 


\section{Data Collection}

At the end of the basketball unit, the participants were organized into four teams of three players. They played two 10 -minute 3 vs. 3 games, which were videotaped by a research assistant. During the videotaping of the games, the research assistant placed the camcorder in an unobtrusive corner of the gymnasium, adjusted the camcorder's angles, and zoomed in and out to ensure that all six players' on-the-ball skills and off-the-ball movements were in view at all times.

Prior to officially coding the two videotaped 10-minute game play sessions, the two investigators spent an estimated 20 hours observing and coding two players' offensive game behaviors with the BOGPI until they were satisfied with the definition of each sub-game component, the rating scales, and the coding protocols. Next, the two investigators independently coded each of the twelve players' on whether they demonstrated the criteria of each sub-game component in the BOGPI when his/her team gained possession of the ball by strictly following the coding protocols. Throughout the two 10-minute game play sessions, a total of 1100 offensive game behaviors were independently coded by the two investigators when their teams were in possession of the ball.

Finally, the coded game performance responses were transformed into an index score of each subgame component on the score sheet. This study used the scoring system proposed by Memmert and Harvey (2008) who used the idea of the $\mathrm{T}$ standard score. In this scoring system, each player started with a score of 10 (a constant for both appropriate/efficient and inappropriate/ inefficient responses). On the score sheet, each time a player demonstrated the criteria of a specific sub-game component, the player gained 1 point in the appropriate/ efficient response column. Similarly, if that player did not demonstrate the criteria of that specific sub-game component, the player gained 1 point in the inappropriate/ inefficient action column. For example, regarding Nancy's dribbling skill execution, she demonstrated the criteria of the Dribbling sub-game component twice (e.g., she was marked as "+" two times on the assessment sheet), so she received 2 points for that appropriate skill execution. In contrast, she did not demonstrate the criteria of the Dribbling sub-game component once (e.g., she was marked as "-" one time on the assessment sheet), so she received 1 point for inappropriate skill execution. Her raw score for appropriate dribbling skill execution would be $2+10=12$, while her raw score for inappropriate dribbling skill execution would $1+10=11$. As Mitchell et al. (2006) suggested, each game component index score was presented as a ratio of appropriate (A)/effective (E) responses to inappropriate (IA) /ineffective (IE) responses, that is, $(\mathrm{A} / \mathrm{E} \div(\mathrm{A} / \mathrm{E}+\mathrm{IA} / \mathrm{IE}))$. Accordingly, her dribbling skill execution index score would be (12 $\div(12+11))=.52$ or $52 \%$ when the percentage is used. Based on Memmert and Harvey's score system (2008), the index score greater than .50 or $50 \%$ and close to 1 or $100 \%$ indicated the player made more appropriate/effective actions than inappropriate/ineffective responses. In contrast, lower than .50 or $50 \%$ meant that a player made more inappropriate/inefficient actions than appropriate/effective actions. The index score of .50 or $50 \%$ indicated a player made the same number of appropriate/effective actions as inappropriate / ineffective responses.

\section{Data Analysis}

To discern content validity of the BOGPI, six pre-service teachers who did not participate in this study were asked to judge the content validity of the instrument. Three male and three female pre-service teachers were selected based on the criteria: (a) playing on varsity basketball team in high school, (b) having at least four years of basketball coaching experiences, (c) either playing on a varsity basketball team in college or participating in basketball intramural/club teams. They were asked to judge whether or not the definition of each subgame component on the BOPGI can best represent on-theball skills (Skill Execution) and off-the-ball movements (Support) and tactical appropriateness of Decision Making in terms of dribbling, passing, and shooting.

To determine the construct validity of the BOGPI, an independent $\mathrm{t}$-test was used to examine if the BOGPI could be used to distinguish the pre-service teachers' game performance ability between the two groups. In this study, the five dependent variables including Skill Execution Index (SEI), Decision Making Index (DMI), Game Performance Index (GPI), and Game Involvement Index (GGI) were used to distinguish higher- and lowerperforming pre-service teachers' game performance ability. Based on the recommendation by Mitchell et al. (2006), each individual game component index score was calculated using the sum of each sub-game component index divided by the number of each sub-game component. Whereas, the GII is the sum of appropriate/ efficient and inappropriate/inefficient responses of each sub- 
game component to reflect the player's total contribution to his/her team's attack.

The inter-rater reliability of the BOGPI was examined by checking each investigator's coding results item by item. To assess the internal consistency reliability of the total scale of the BOGPI, Cronbach alpha reliability coefficient was used to analyze the data of 1100 offensive game responses.

\section{Results}

\section{Validity of the BOGPI}

Content validity. To determine the content validity of the BOGPI, the six pre-service teachers who met the criteria stated above were selected as a panel of experts. They were provided with the questionnaire, which consisted of the sub-game components under each essential game dimensions of Skill Execution, Decision Making, and Support in the BOGPI, a five-point rating scale anchored with 1 (does not precisely) to 5 (very precisely), and an open comments/edits section. With respect to the Skill Execution dimension, the experts rated the definition of dribbling and passing very precisely (66\% rated on 5) and precisely (33\% rated on 4 point) stated and reflected the game dimension; they rated the definition of shooting precisely (50\% rated on 4 point) and sort of precisely (50\% rated on 3 point) stated and reflected the game dimension. Three experts suggested changing the original definition of shooting (i.e., shoots when appropriate and scores basket) with the new definition of "shoots when getting open and scores a basket." The new definition suggested by the experts was adopted in the BOGPI. Regarding the Decision Making dimension, the experts rated that the definitions of the three sub-game components very precisely (66\% rated on 5 point) and precisely (34\% rated 4 on point) stated and reflected the game dimension. They did not make any edits for the definitions. With regards to the Support dimension, the experts rated the definitions of the four sub-game components very precisely (50\% rated on 5 point) and precisely (50\% rated on 4 point) stated and reflected the game dimension. No edits were made. The experts' judgment results indicated that the content validity of the BOGPI was established.

Construct validity. To examine the construct validity of the BOGPI, the mean score of GPI was used to divide the participants into two groups because GPI provides a comprehensive view of a player's game performance competency (Mitchell et al., 2006). The mean score of the GPI was .55. The participants' GPI scores greater than .55 were classified into the highgame performance, while the others were in the low-game performance group. Accordingly, seven participants were in the high-game performance group and five participants were in the low-game performance group. Table 2 presents the descriptive statistics of the Skill Execution Index (SEI), the Decision Making Index (DMI), the Support Index (SI), the Game Involvement Index (GII), and the Game Performance Index (GPI) between the two groups.

Table 2. Descriptive Statistics of the Coded Offensive Game Reponses between the Two Groups.

\begin{tabular}{|c|c|c|c|c|c|c|c|c|c|c|}
\hline & SEI & & DMI & & SI & & GPI & & GI & \\
\hline & $M$ & $S D$ & $M$ & $S D$ & $M$ & $S D$ & $M$ & $S D$ & $M$ & $S D$ \\
\hline Low group & .51 & .014 & .55 & .038 & .54 & .019 & .53 & .023 & .35 & .056 \\
\hline High group & .55 & .017 & .59 & .016 & .57 & .014 & .57 & .010 & .46 & .078 \\
\hline
\end{tabular}

The independent t-test revealed a significant difference in the mean scores of the GII between the low- and the high-game involvement groups $(\mathrm{t}=-2.837$, $\mathrm{p}<.01)$. The results indicated that BOGPI provided discernable information about the overall game involvement between the two groups. Similarly, the independent t-test yielded that the mean score of the GPI in the low-game performance group was significantly lower than that of the GPI in the high-game performance group $(\mathrm{t}=-3.928$, $p<.01)$. The result of the $t$-test indicated that the 
BOGPI was a valid instrument to distinguish the players' overall offensive game ability between low- and high-game performance groups.

With regards to the SEI, the independent t-test indicated that the low-game performance group scored significantly lower than the high-game performance group (t $=-4.786, \mathrm{p}<.01)$. Regarding the DMI, the $\mathrm{t}$-test yielded a difference of the mean scores between the low-game performance group $(\mathrm{t}=-2.112, \mathrm{p}=.06)$. With respect to the SI, the t-test indicated a significant difference $(\mathrm{t}=$ $-3.859, \mathrm{p}<.01$ ) between the low-game performance group. The results indicated that the three individual game dimensions on the BOGPI could be used to differentiate the players' ability of executing skills, making decisions, and providing support between the low- and high-game performance groups.

\section{Reliability of the BOGPI}

Among the total coded 1100 game responses, the number of agreement was 1089, while the number of disagreements was 11. According to the formula (IR\% $=1089 \div(1089+11))$, the inter-rater reliability of the BOGPI was 99\%, indicating a high consistency of two raters' judgment (van der Mars, 1989). The alpha reliability coefficient for the total scale of the BOGPI was .95, higher than .70 (Stevens, 2002). The results showed that the BOGPI had a high degree of internal consistency reliability (Stevens, 2002).

\section{Discussion}

The content validity of the BOGPI was established by determining whether or not the instrument was designed to assess what it purported to measure. The BOGPI was designed to assess pre-service teachers' offensive game performance competency in basketball. The three essential dimensions of the BOGPI including Skill Execution, Decision Making, and Support were consistently viewed as critical game components to assess offensive game performance in the invasion game form (Oslin et al., 1998). The three dimensions of the BOGPI provided adequate opportunities for the players to demonstrate offensive game behaviors during basketball game play. The definition of the sub-game components specified how effectively the player applied on-the-ball skills and offthe-ball movements appropriate to specific game situations. The sub-game components were essential and relevant to measuring the given game dimension of the BOGPI.
The construct validity of the BOGPI was established in this study. The results of the t-tests indicated that the BOGPI was a valid instrument to differentiate the players' overall game performance and game involvement levels between the high- and low-game performance groups. Furthermore, this study indicated that the Skill Execution, Decision Making, and Support were valid individual game performance variables to distinguish the players' offensive game ability in basketball between the two groups. Similar to the results of this study, Oslin et al. (1998) reported the significant differences in the Skill Execution, Decision Making, and Support between the high- and low- game performance groups in their study of basketball. This study suggested that the players in the high-game performance group demonstrated more appropriate responses in terms of the effective execution of the dribbling, passing, and shooting skills, choosing the skills with tactical purposes, and using of the off-the-ball movements to accommodate particular game situations. In contrast, the players in the low-game performance group demonstrated less effective use of the dribbling, passing, and shooting skills. They attempted to choose the skills with a lack of tactical purposes.

The results of the inter-rater reliability of the BOGPI (99\%) revealed that two independent evaluators coded a total of 1100 game behavior occurrences with a very high degree of consistency. The specific definition of each sub-game component, the event recording method, and the coding protocols might collectively contribute to this promising result. First, the definition of each sub-game component provided articulated guidelines for evaluators to understand what specific game performance behaviors they should focus on observing when a player is with and without the ball. Second, the event recording method in the BOGPI helped the evaluators objectively code whether or not the pre-service teacher demonstrated the criteria of each subgame component within the respective column. Third, the specific coding protocols provided the evaluators with specific guidelines in terms of whom to observe at a time and when to switch to observation of another player. In conclusion, this study suggests that the BOGPI is a theoretically sound and psychometrically supported measure. It can be used for researchers and teacher educators to assess the pre-service teachers' offensive game performance ability in basketball using videotaped game play. Future studies may use broad samples of pre-service teachers in various PETE programs to examine the psychometric properties within the cross-sectional and/or longitudinal research designs. 


\section{References}

Gréhaigne, J. F., Godbout, P., \& Bouthier D. (2001). The teaching and learning of decision making in team sports. Quest, 53, 59-76.

Kirk, D., \& MacPhail, A. (2002). Teaching games for understanding and situated learning: Rethinking the Bunker-Thorpe Model. Journal of Teaching in Physical Education, 21, 177-192.

Lave, J., \& Wenger, E. (1991). Situated learning: Legitimate peripheral participation. New York: Cambridge University Press.

McCullick, B. A. (2001). Practitioners' perspectives on values, knowledge, and skills needed by PETE participants. Journal of Teaching in Physical Education, 21, 35-56.

Memmert, D., \& Harvey, S. (2008). The game performance assessment instrument (GPAI): some concerns and solutions for further development. Journal of Teaching in Physical Education, 27, 220240.

National Association for Sport and Physical Education (NASPE) (2009). National standards \& guidelines for physical education teacher education (3rd ed.). Champaign, IL: Human Kinetics.

Oslin, J. L., Mitchell, S. A., \& Griffin, L. L. (1998). The game performance assessment instrument (GPAI): Development and preliminary validation. Journal of Teaching in Physical Education, 17, 231-243.

Rovegno, I. (2006). Situated perspectives on learning. In D. Kirk, D. Macdonald, \& M. O'Sullivan (Eds.), The Handbook of Physical Education (pp. 262-264). London, UK: Sage Publications.

Siedentop, D. (2002). Content knowledge for physical education. Journal of Teaching in Physical Education, $21,368-377$.

Stevens, J. (2002). Applied multivariate statistics for the social sciences $\left(4^{\text {th }}\right.$ ed.). Hillsdale, NJ: Lawrence Erlbaum. van der Mars, H. (1989). Observer reliability: Issues and procedures. In P. Darst, D. Zakrajsek, \& V. Mancini (Eds.), Analyzing physical education and sport instruction $\left(2^{\text {nd }}\right.$ ed., pp. 53-80). Champaign, IL: Human Kinetics.

\section{Correspondence}

Weiyun Chen, Ph.D

School of Kinesiology, University of Michigan, Observatory Lodge: 3145, 1402 Washington Hts. Ann Arbor, MI 48168-2013

Phone: 734-615-0376; Fax: 734-647-2802

Email: chenwy@umich.edu 